\section{PTH-002 COLONOSCOPY AFTER BOWELSCOPE (BS) FLEXIBLE SIGMOIDOSCOPY (FS) - URGENT OR ROUTINE?}

${ }^{1}$ Anwar Suleiman Abusrewil ${ }^{*},{ }^{1}$ Mumtaz Hayat, ${ }^{2}$ Heather Dixon, ${ }^{2}$ Karen Rowell, ${ }^{3}$ David Nylander. ${ }^{1}$ Northumbria NHS Healthcare Trust, North Shields, UK; ${ }^{2}$ North of Tyne Screening Centre, Newcastle upon Tyne, UK; ${ }^{3}$ Newcastle upon Tyne Foundation Trust, Newcastle upon Tyne, UK

\subsection{6/gutjnl-2018-BSGAbstracts.24}

Introduction and aims The implementation of BS (1 off FS for 55 year olds) has led to significant pressures on endoscopy units with large numbers of procedures. In addition patients with certain findings (which include number, size, dysplasia and villous component degree of adenomatous polyps) will require colonoscopy (FC). This has to be done within 2 weeks. The North of Tyne screening centre serves a population >8 60 000. 'Roll out' of BS started in 2014 and now covers patients enrolled in $50 \%$ of our regional GP practices.

At this 'halfway stage' we aimed to assess:

- Attendance and findings in those invited

- Proportion of patients who require FC after FS and significance of proximal pathology

- Incidence and sites of any malignancy

- The proportion with neoplasia after colonoscopy requiring future surveillance

Method Data was collected on all patients who had FC after FS in the BS program for the 12 months from 1/1/2017 (obtained from the central database and crosschecked with local records). We reviewed all endoscopy and histology reports to obtain patient demographics, FC indication, findings and all histology. The extent of each FS was accurately recorded with aid of Olympus imager (scope guide).

Results 2698 of the 3629 who responded to the written invitation attended for FS.

130 (4.8\% of attenders) met criteria for FC - Main reasons: $\geq 10 \mathrm{~mm}$ polyp $(34 \%) ; \geq 3$ polyps $(21.5 \%)$; villous histology $(21.5 \%)$; anticoagulant/antiplatelet use $(4.6 \%)$

After colonoscopy, 54 have neoplasia requiring for future surveillance -33 high risk category (1 year); 21 , intermediate risk (3 years).

4 patients had malignancies: $1 \mathrm{x}$ rectal polyp cancer; $1 \mathrm{x}$ sigmoid cancer (T2N0); $1 \mathrm{x}$ descending colon cancer (T3N1M1); 1 splenic flexure cancer (T4N1);

At colonoscopy, 37 patients had adenomas proximal to the splenic flexure but all were $<10 \mathrm{~mm}$ with low-grade dysplasia Conclusions

- 74\% of patients who initially showed interest attended for FS

- Almost 5\% of patients attending for BS require FC; of these $41.5 \%$ will have intermediate or high risk neoplasia requiring future surveillance

- A small proportion (1.5/1000 screened) of attenders were found to have a cancer

- 28.5\% had neoplastic lesions beyond the splenic flexure, none with high grade dysplasia/cancer

Therefore if the colon is examined to the splenic flexure at FS during BS screening, our data suggests that FC can be safely booked as routine (within 6 weeks). This will ease some of the pressure on endoscopy units.
PTH-003 QIP: IMPROVING QUALITY OF PHOTODOCUMENTATION AND LESION REPORTING IN UPPER GASTROINTESTINAL ENDOSCOPY

${ }^{1}$ Ahmir Ahmad*, ${ }^{1} J u l i e t$ Bottle, ${ }^{2}$ Anthony Laverty, ${ }^{1}$ Sam Murray, 'lain Ewing. ${ }^{1}$ Homerton University Hospital, London, UK: ${ }^{2}$ Imperial College London, London, UK

\subsection{6/gutjnl-2018-BSGAbstracts.25}

Introduction The gold standard investigation for upper gastrointestinal (GI) symptoms is oesophago-gastro-duodenoscopy (OGD). The British Society of Gastroenterology (BSG) released a position statement of quality standards in upper gastrointestinal endoscopy in August 2017, including the recommendation to photodocument 8 anatomical sites in the upper GI tract to optimise mucosal inspection and lesion recognition. We aimed to assess and improve the quality of OGD photodocumentation and lesion recognition in line with this recommendation.

Methods 184 OGD reports were audited from three one-week audit cycles. Cycle 1: immediately following guideline release and prior to intervention; cycle 2: immediately following intervention (departmental teaching and poster display of recommended photos in endoscopy procedure rooms); cycle 3: 2 weeks after intervention. Total number of photos taken per procedure, number of anatomical sites photodocumented, and number of lesions detected were recorded.

Results From cycle 1 (pre-intervention) to cycle 2 (post-intervention) the mean number of photos per OGD increased from 5.3 to 8.6 respectively $(p=0.095)$ and mean number of anatomical sites documented increased from $3.8(47 \%)$ to 6.9 $(86 \%) \quad(p<0.001)$. The mean number of lesions documented per OGD increased from 0.5 to $1.2(p=0.230)$. Improvement in practice was sustained through audit cycle 3 (2 weeks after intervention): mean photos 8.5, mean required sites documented $7.4(92 \%)$ and 0.7 diagnoses per OGD.

Conclusions Immediately following release of BSG quality standards in upper GI endoscopy, departmental adherence to recommendations for photodocumentation was poor. A simple intervention of departmental teaching and poster displays resulted in a significant improvement in the quality of photodocumentation and a trend towards improvement in detection of lesions. Improvement in quality was sustained at re-audit.

\section{PTH-004 DEEP LEARNING FOR REAL-TIME AUTOMATED POLYP LOCALISATION IN COLONOSCOPY VIDEOS}

${ }^{1}$ Omer F Ahmad", ${ }^{1}$ Imanol Luengo, ${ }^{1}$ Luis Garcia Peraza Herrera, ${ }^{1}$ Patrick Brandao, ${ }^{1}$ Wenqi Li, ${ }^{2}$ Martin Everson, ${ }^{2}$ Rehan Haidry, ${ }^{2}$ Roser Vega, ${ }^{2}$ Ed Seward, ${ }^{1}$ Tom Vercauteren, 1,2 Laurence B Lovat. 'Wellcome/EPSRC Centre for Interventional and Surgical Sciences, University College London; ${ }^{2}$ Gastrointestinal Services, University College London Hospital

\subsection{6/gutjnl-2018-BSGAbstracts.26}

Introduction Colonoscopic polypectomy can prevent colorectal cancer. Polyp detection rates vary considerably due to human error and missed adenomas may contribute to interval colorectal cancers. Automated polyp detection using deep learning may avoid these problems. Previous work focused on detecting the presence of polyps in individual frames captured from videos. Our aims in this pilot study were to extend this to video 